\title{
Cardiac pacing: A novel approach to right ventricle failure during pulmonary thromboendarterectomy
}

\author{
Vinod Havalad, MD, ${ }^{\mathrm{a}}$ Matthew Bacchetta, MD, ${ }^{\mathrm{b}}$ Daniel Y. Wang, MD, ${ }^{\mathrm{c}}$ Santos E. Cabreriza, MBA, ${ }^{\mathrm{b}}$ \\ Linda Aponte-Patel, MD, ${ }^{\mathrm{a}}$ Bin Cheng, PhD, ${ }^{\mathrm{d}}$ and Henry M. Spotnitz, MD, ${ }^{\mathrm{b}}$ Park Ridge, Ill, and New York, NY
}

Pulmonary thromboendarterectomy (PTE) reverses chronic thromboembolic pulmonary hypertension, decreasing pulmonary vascular resistance and pressure overload failure of the right ventricle (RV). Suboptimal cardiac function early after $\mathrm{PTE}^{1}$ on cardiopulmonary bypass $(\mathrm{CPB})$ may require inotropic or mechanical support. Our laboratory demonstrated increased cardiac output $(\mathrm{CO})$ with temporary biventricular pacing (BiVP) in an RV pressure overload pig model. ${ }^{2}$ In patients with left ventricular (LV) dysfunction, optimized temporary BiVP increases $\mathrm{CO}$ after $\mathrm{CPB},{ }^{3}$ reduces the vasoactiveinotrope score, and increases urine output. ${ }^{4}$ Our study tests the feasibility of BiVP reversal of RV failure after PTE.

\section{MATERIALS AND METHODS}

Four adults were recruited and studied with informed consent under an Institutional Review Board-approved protocol between June 1, 2011, and March 1, 2012. Inclusion criteria were PTE on CPB, sinus rhythm (NSR), and inotrope dependence after CPB. Exclusion criteria were atrial fibrillation, $2^{\circ}$ or $3^{\circ}$ atrioventricular block, congenital heart disease, intracardiac shunts, inability to pace or place an ultrasonic aortic flow probe (UFP), or heart rate $(\mathrm{HR})>120 \mathrm{bpm}$ after $\mathrm{CPB}$. Our surgeon performed PTE using standardized methods. ${ }^{5}$ There were no study-related complications.

This protocol resembles phase I of the Biventricular Pacing after Cardiac Surgery trial. ${ }^{3}$ Temporary epicardial pacing wires sewn to the appendage of the right atrium (RA), anterior RV, and LV obtuse margin were connected to an InSync III pacemaker (Medtronic Inc, Minneapolis, Minn) for BiVP. A UFP device (Transonic Systems, Ithaca, NY) was used to measure CO.

The protocol begins after weaning from CPB and hemodynamic stabilization. Pacing rate is $90 \mathrm{bpm}$ or $10 \mathrm{bpm}$ higher than intrinsic HR if $>90$ bpm. Seven values of atrioventricular delay (AVD) are tested (90 to 270 milliseconds; 30-millisecond increments). Nine values of interventricular delay (VVD) are also tested ( -80 millisecond $[\mathrm{LV}$ first $]$ to +80 millisecond [RV first]; 20-millisecond increments). Settings are tested twice for 10 seconds in random sequence. Optimum AVD and VVD are selected using a graphical display. Vasoactive medications and volume infusion are held constant during optimization. Finally, optimized BiVP, atrial pacing

From the Departments of Pediatrics, ${ }^{\text {a }}$ Advocate Children's Hospital, Park Ridge, Ill; and Departments of Surgery, ${ }^{\mathrm{b}}$ and Medicine, ${ }^{\mathrm{c}}$ and Mailman School of Public Health, ${ }^{\mathrm{d}}$ Columbia University, New York, NY.

Supported in part by a Startup Grant from the Department of Surgery, Columbia University, New York, NY.

Disclosures: Authors have nothing to disclose with regard to commercial support.

H.M.S. is George H. Humphreys, II, Professor of Surgery, Columbia University, New York, NY.

Received for publication May 9, 2012; revisions received Dec 12, 2012; accepted for publication Dec 18, 2012; available ahead of print Jan 14, 2013.

Address for reprints: Henry M. Spotnitz, MD, Department of Surgery, Columbia University Medical Center, $622 \mathrm{~W}$ 168th St, 10th Fl, Vanderbilt Clinic, Rm 1010, New

York, NY 10032 (E-mail: hms2@ columbia.edu).

J Thorac Cardiovasc Surg 2013;145:1141-3

$0022-5223 / \$ 36.00$

Copyright $\odot 2013$ by The American Association for Thoracic Surgery

http://dx.doi.org/10.1016/j.jtcvs.2012.12.068

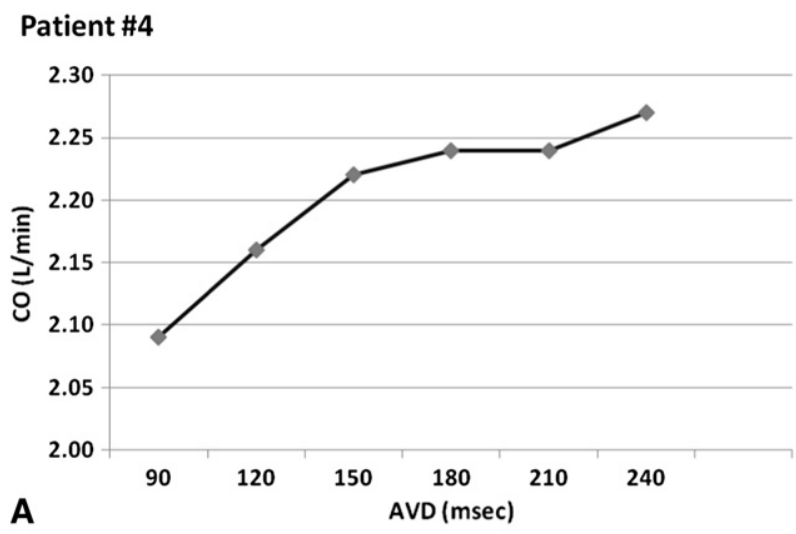

\section{Patient \#4}

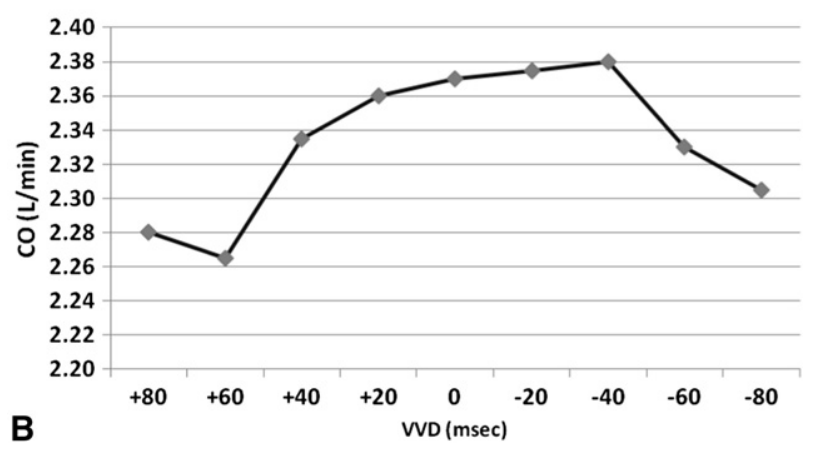

FIGURE 1. A, Representative effect of atrioventricular delay ( $A V D)$ optimization on cardiac output $(C O)$. B, Representative effect of interventricular delay $(V V D)$ optimization on $\mathrm{CO}$.

(AAI), and NSR are tested twice in randomized order over 30-second intervals. The UFP and LV wires are then removed. Paired $t$ tests are used to compare NSR to optimized BiVP and AAI.

\section{RESULTS}

Representative AVD optimization is illustrated in Figure 1, A, CO peaking at 240 milliseconds. During VVD optimization, CO peaks at -40 milliseconds (LV pacing 40 milliseconds before RV pacing), as shown in Figure $1, B$. The change in CO is $<1 \%$ between VVDs of +20 and -40 milliseconds.

Table 1 illustrates clinical and optimization data for each patient. AVD optima were 120 to 270 milliseconds. VVD optima were $-40,0,0$, and 60 milliseconds. All patients were in RV failure preoperatively and were receiving inotropic support at the time of testing.

Figure 2 compares mean $\mathrm{CO}(\mathrm{n}=4)$ during AAI, BiVP, and NSR. Mean \pm standard deviation $\mathrm{CO}$ during AAI 
TABLE 1. Clinical and optimization data for each patient $(\mathbf{N}=4)$

\begin{tabular}{|c|c|c|c|c|c|c|c|c|c|c|c|c|c|c|}
\hline & & PAP s/d/m & QRSd & & & $\mathrm{CA}$ & $\mathrm{XC}$ & CPB & BSA & AVD & VVD & NoP & AAI & $\mathrm{BiV}$ \\
\hline Patient & $\mathbf{A} / \mathbf{G}$ & $\mathbf{m m ~ H g}$ & msec & RBBB & RVH & h:m & h:m & h:m & $\mathbf{m}^{2}$ & msec & msec & $\mathrm{CO} \Delta$ & $\mathrm{CO} \Delta$ & $\mathrm{CO} \Delta$ \\
\hline PTE001 & $54 \mathrm{~m}$ & $68 / 34 / 54$ & 168 & No & Yes & 57 & $1: 21$ & $4: 10$ & 2.1 & 270 & 0 & 0 & 3.1 & -5.6 \\
\hline PTE002 & $31 \mathrm{~m}$ & $99 / 21 / 44$ & 192 & No & No & 1:02 & $1: 36$ & $5: 27$ & 2.4 & 180 & 60 & 0 & 10.7 & 3 \\
\hline PTE003 & $66 f$ & $64 / 33 / 44$ & 154 & No & Yes & 40 & 56 & $3: 55$ & 1.8 & 120 & 0 & 0 & 6.5 & 1.2 \\
\hline PTE004 & $45 \mathrm{f}$ & $96 / 28 / 55$ & 128 & Yes & No & 39 & $1: 39$ & 5:07 & 2.2 & 180 & -40 & 0 & 12.6 & 11.5 \\
\hline
\end{tabular}

Minimum temperature all cases was $18^{\circ} \mathrm{C} . A / G$, Age/gender; $P A P$, pulmonary artery pressure; $s / d / m$, systolic/diastolic/mean; $Q R S d$, QRS duration before $\mathrm{CPB} ; R B B B$, right bundle branch block; $R V H$, right ventricular hypertrophy; $C A$, circulatory arrest; $X C$, crossclamp; $C P B$, cardiopulmonary bypass; $B S A$, body surface area; $A V D$, atrioventricular delay; $V V D$, interventricular delay; $N o P$, no pacing; $A A I$, atrial pacing; $B i V$, biventricular pacing; $h: m$, hours:minutes; $C O \Delta$, percent change in cardiac output vs AAI; $m$, male; $f$, female.

was $8.4 \% \pm 3.8 \%$ higher than in NSR $(P=.04)$. CO during BiVP was not significantly different versus NSR. Mean \pm standard deviation HR during BiVP and AAI was $94.0 \pm 4.7 /$ minute versus $76.3 \pm 6.9 /$ minute during NSR $(P<.01)$.

\section{DISCUSSION}

This is a novel pilot study of the hemodynamics of BiVP during PTE. As in the Biventricular Pacing after Cardiac Surgery trial, ${ }^{3}$ our results demonstrate that AVD and VVD optimization increase $\mathrm{CO}$ during BiVP. However, $\mathrm{CO}$ during AAI in our study was significantly higher than with both BiVP and NSR. CO after CPB in the Biventricular Pacing after Cardiac Surgery trial was $10 \%$ to $13 \%$ higher with optimized BiVP than with NSR or AAI.

Minimal efficacy of optimized BiVP in our study contrasts with recent catheter lab research in patients with chronic thromboembolic pulmonary hypertension. ${ }^{6}$ Additional research is needed to determine if our results are an anomaly, related to a small patient cohort. Dual-site RV pacing is a possible alternative approach, particularly in patients with right bundle branch block.

Ventricular mechanics analysis might explain the reduced efficacy of BiVP observed here. The time course of pulmonary and systemic arterial pressure might help, as suggested by laboratory studies utilizing RV and LV pressure and 2-dimensional echocardiography. ${ }^{2}$ Heart block in those studies prevented comparison with AAI, but BiVP was effective at the intrinsic atrial rate. ${ }^{2}$

We attribute BiVP efficacy in the Biventricular Pacing after Cardiac Surgery trial to reversal of ischemia-reperfusion injury. Such injury may have been minimized in our study by the long rewarming time. With no clear BiVP benefit, HR augmentation may be the only potential advantage of pacing after PTE. Clinical trials are needed to define benefits and problems with pacing in critically ill patients with PTE, although temporary HR augmentation is apparently recommended in some centers after CPB.

As in the Biventricular Pacing after Cardiac Surgery trial, the optimum AVD noted here for BiVP is often considerably longer than in "permanent" pacemakers or BiVP implants.

\section{CONCLUSIONS}

AAI improves CO, and temporary BiVP is feasible after PTE. However, BiVP was minimally effective in this series. Temporary pacing warrants further study to define the full spectrum of response after PTE.

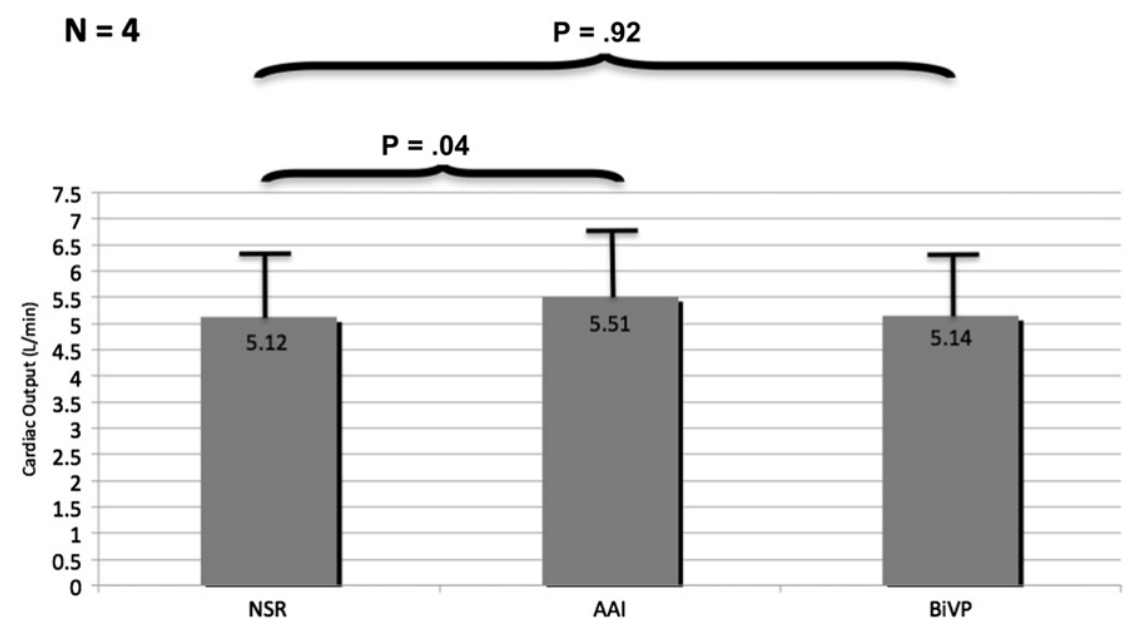

FIGURE 2. Comparison of cardiac output $(C O)$ in sinus rhythm $(N S R)$ versus atrial pacing $(A A I)$ or optimized biventricular pacing $(B i V P)$ in 4 patients. Bars indicate standard error of the mean and $P$ values are based on paired $t$ test. 


\section{References}

1. D'Armini AM, Zanotti G, Ghio S, Magrini G, Pozzi M, Scelsi L, et al. Reverse right ventricular remodeling after pulmonary endarterectomy. $J$ Thorac Cardiovasc Surg. 2007;133:162-8.

2. Rabkin DG, Cabreriza SE, Curtis LJ, Mazer SP, Kanter JP, Weinberg AD, et al. Load dependence of cardiac output in biventricular pacing: right ventricular pressure overload in pigs. $J$ Thorac Cardiovasc Surg. 2004;7:1713-22.

3. Wang DY, Richmond ME, Quinn TA, Mirani AJ, Rusanov A, Yalamanci V, et al. Optimized temporary biventricular pacing acutely improves intraoperative cardiac output after weaning from cardiopulmonary bypass: a substudy of a randomized clinical trial. J Thorac Cardiovasc Surg. 2011;141: 1002-8
4. Nguyen HV, Havalad V, Aponte-Patel L, Wang DY, Cheng B, Cabreriza SE, et al. Temporary biventricular pacing decreases the vasoactive-inotropic score after cardiac surgery: a substudy of a randomized clinical trial. J Thorac Cardiovasc Surg. July 27, 2012 [Epub ahead of print].

5. Thistlethwaite PA, Kaneko K, Madani MM, Jamieson SW. Technique and outcomes of pulmonary endarterectomy surgery. Ann Thorac Cardiovasc Surg. 2008; 14:274-82.

6. Hardziyenka M, Surie S, de Groot JR, de Bruin-Bon HA, Knops RE Remmelink M, et al. Right ventricular pacing improves haemodynamics in right ventricular failure from pressure overload: an open observational proof-ofprinciple study in patients with chronic thromboembolic pulmonary hypertension. Europace. 2011;13:1753-9. 